\title{
Relación de recuento de leucocitos con desviación a la izquierda y el hallazgo quirúrgico en apendicitis aguda en el servicio de urgencias adulto del Hospital de Clínicas en el periodo 2015 al 2019
}

\author{
Relationship of leukocyte count with left deviation and surgical finding in \\ acute appendicitis in the adult emergency department of the Hospital of \\ Clinics in the period 2015 to 2019
}

\author{
Cristhian Chavez Rivaldi ${ }^{\circledR}$, Rosana Edith Godoy Ruiz Díaz ${ }^{2}$, Rodrigo Amarilla Larrieur ${ }^{3}$, \\ Rafael Acosta Fernández $z^{4}$
}

Universidad Nacional de Asunción Facultad de Ciencias Médicas - Hospital de Clínicas - Servicio de Urgencias de Cirugía General. San Lorenzo, Paraguay

\begin{abstract}
RESUMEN
La apendicitis es la inflamación del apéndice ileocecal. Pacientes y métodos: 892 casos de Apendicitis Aguda sometidos a cirugía en el servicio de Urgencias Adultos del Hospital de Clínicas San Lorenzo entre los años 2015 al 2019, trabajo retrospectivo, descriptivo, transversal que tiene como objetivo investigar la relación del recuento de leucocitos con desviación a la izquierda y el hallazgo quirúrgico. Resultado: En un 77,6\% el conteo de glóbulos blancos fue igual o mayor a 11.000 ,

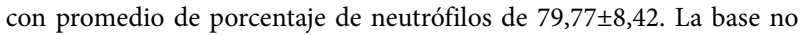
comprometida se encontró en $96 \%$ de los casos. El hallazgo quirúrgico más frecuente fue la Gangrenosa (37,7\%). Conclusión: Evidenciamos presencia de leucocitosis en un $77 \%$ relacionado a apendicitis aguda.

Palabras clave: apendicitis, dolor fosa iliaca derecha, leucocitosis.
\end{abstract}

\section{ABSTRACT}

Appendicitis is the inflammation of the ileocecal appendi. Patients and methods: 892 cases of Acute Appendicitis undergoing surgery in the Adult Emergency Service of the Hospital de Clínicas San Lorenzo between the years 2015 and 2019, retrospective, descriptive, cross-sectional work that aims to investigate the relationship of the leukocyte count with deviation on the left and the surgical finding. Result: A 77.6\% was found in terms of the white blood cell count equal to or greater than 11,000 , with an average percentage of neutrophils of $79,77 \pm 8,42$. The uncompromised base was found in $96 \%$ of the cases. The most frequent surgical finding was gangrenous with $37.7 \%$. Conclusion: We evidenced the presence of leukocytosis in $77 \%$ related to acute appendicitis.

Key Words: appendicitis, right iliac fossa pain, leukocytosis.

\section{INTRODUCCIÓN}

La palabra "Apendicitis" es un vocablo derivado del latín apendix (apéndice) y del sufijo griego itis (inflamación). Etimológi- camente significa inflamación del apéndice ileocecal ${ }^{(1)}$. Dentro del cuadro de abdomen agudo quirúrgico, la apendicitis aguda es una de las patologías más frecuentes que admite pacientes a los servicios de urgencias de diferentes hospitales ${ }^{(2)}$. Se presenta aproximadamente en el 10\% de la población general y existe un pico de máxima incidencia entre los 10 y los 20 años de edad ${ }^{(3)}$. Fitz idéntico la apendicitis como entidad clínica y patológica en el año 1886, más adelante, Mc Burney describió las manifestaciones clínicas tempranas de dicha patología antes de su perforación $^{(4)}$. Hoy en día, el cuadro de apendicitis aguda es una condición bastante frecuente y un diagnóstico diferencial muy común en pacientes que presentan dolor abdominal agudo. El diagnóstico es a menudo elusivo y el manejo de pacientes con diagnósticos equivocados es controversial ${ }^{(5)}$. En Estados Unidos ocurren 250000 casos por año, estimándose en 1000000 los días de hospitalización y 1,5 billones de dólares anuales gastados en el diagnóstico y tratamiento. La incidencia es de 11 casos por 10000 personas/año y se incrementa entre los 10 y 20 años de edad a 23 por 10000 personas/año ${ }^{(6)}$. En los últimos años se ha intentado instaurar un abordaje diagnóstico tecnológico, que incluye del uso de las denominadas puntuaciones diagnósticas, estudios imagenológicos especializados, exámenes complementarios de laboratorio, como el recuento de leucocitos (RL) y la concentración plasmática de proteína C reactiva (PCR) que ha sido extensamente estudiada en el diagnóstico de apendicitis con resultados variados y contradictorios ${ }^{(7)}$. Los estudios

1. Autor principal. Residente $1^{\circ}$ año Sala X. Hospital de Clínicas FCM-UNA. Paraguay

2. Residente $3^{\circ}$ año Sala X. Hospital de Clínicas FCM-UNA. Paraguay

3. Especialista en Cirugía General, Subjefe de Urgencias Cirugía General del Hospital de Clínicas San Lorenzo

4. Especialista en Cirugía General, Jefe de Sala de la Primera Cátedra de Clínica Quirúrgica del Hospital de Clínicas San Lorenzo

Autor Correspondiente: Dr. Cristhian Chavez Rivaldi. Hospital de Clínicas. San Lorenzo, Paraguay. Email: chavezrivaldi@outlook.com

Rafael Acosta Fernández, Jefe de Sala de la Primera Cátedra de Clínica Quirúrgica - Sala X del Hospital de Clínicas. racosta@med.una.py

Recibido: 12/09/2020 - Aceptado: 02/10/2020

Este es un artículo publicado en acceso abierto bajo una licencia Creative Commons 
de imagen agregan costo a la hospitalización, no se encuentran disponibles en todos los hospitales y no siempre aumentan en forma significativa la precisión diagnóstica ${ }^{(8)}$.

Actualmente, la decisión del tratamiento quirúrgico de la apendicitis aguda sigue basándose en la evolución del cuadro clínico con sus manifestaciones clásicas de dolor abdominal localizado en fosa ilíaca derecha (FID) con signo de Mc Burney positivo, signo de Blumberg positivo, entre otros. Las pruebas clásicas de laboratorio clínico como el recuento de leucocitos y su fórmula, todavía no están bien entendidas, ya que estas se presentan aumentadas en un alto porcentaje de casos de apendicitis aguda confirmada.

\section{MATERIALES Y MÉTODOS}

Estudio de tipo observacional no experimental, transversal, retrospectivo. Muestreo no probabilístico de casos consecutivos. Revisión de historias clínicas del servicio de Urgencias Adultos de Cirugía General del Hospital de Clínicas de la ciudad de San Lorenzo, Paraguay entre el periodo de 2015 al 2019.La serie incluyen 892 pacientes operados por apendicitis aguda en el servicio, el 49,5\% (441) de los pacientes es de sexo femenino y el $50,5 \%$ (451) de sexo masculino (Tabla 1), la edad promedio fue de $35,75 \pm 17,40$ años, viendo que el $50 \%$ delos pacientes tiene 33 años o menos, observándose un mínimo de 15 años y un máximo de 96 (Figura 1).

Tabla 1. Fuente: Base de datos del servicio de Urgencias Adultos de Cirugía del Hospital de Clínicas

\begin{tabular}{|l|l|l|}
\hline SEXO & FRECUENCIA & PORCENTAJE \\
\hline Femenino & 442 & 49,5 \\
\hline Masculino & 450 & 50,4 \\
\hline Total & 892 & 100 \\
\hline
\end{tabular}

\section{Criterio de inclusión}

Se incluyeron aquellas fichas completas de pacientes con diagnóstico de apendicitis aguda en el servicio de Urgencias Adultos del Hospital de Clínicas de la ciudad de San Lorenzo.

\section{Variables}

- Motivo de consulta

- Horas de evolución

- Recuento de glóbulos blancos

- Recuento de neutrófilos

- Incisión quirúrgica

- Hallazgo quirúrgico

- Base apendicular comprometida o no

\section{RESULTADOS}

Los motivos de consulta más frecuentes fueron el dolor en fosa iliaca derecha con el 53,04\% (847) y el dolor en epigastrio con el $30,12 \%$ (481), sumando entre ambos el 83\% (1328) del total de casos estudiados. Los siguientes motivos en orden de frecuencia fueron; dolor periumbilical con el 8,08\% (129), dolor en hipogastrio con un 4,51\% (72), dolor en flanco derecho 3,63\% (58), dolor en fosa iliaca izquierda $0,56 \%$ (9) y Dolor abdominal generalizado 0,06\% (1) (Figura 2).

Podemos observar que el promedio de horas de evolución en los pacientes fue de $32,52 \pm 20,89$, teniendo el $50 \%$ de los pacientes 24 horas de evolución o menos. Se vio un mínimo de 3 horas y un máximo de 96 (Figura 3).
En los pacientes estudiados se pudo constatar un 77,6\% (690) un conteo de glóbulos blancos iguales o mayores a 11.000 (Figura 4), habiendo un promedio de porcentaje de neutrófilos de $79,77 \pm 8,42$, siendo el $50 \%$ de las muestras iguales o menores a 80 porciento. El mínimo fue de 50\% y el máximo de 97 (Figura 4).

En cuanto a la incisión quirúrgica implementada por el equipo de cirugía, se observó que en el 68,7\% (613) se utilizó la

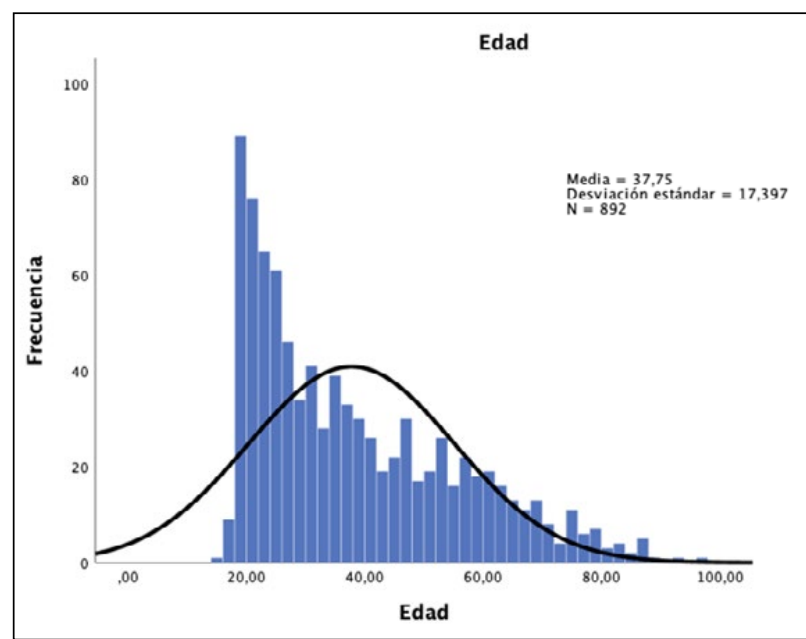

Figura 1. Distribución de la edad de pacientes operados por apendicitis aguda entre los años 2015-2019 en el servicio de Urgencias Adultos de Cirugía del Hospital de Clínicas.

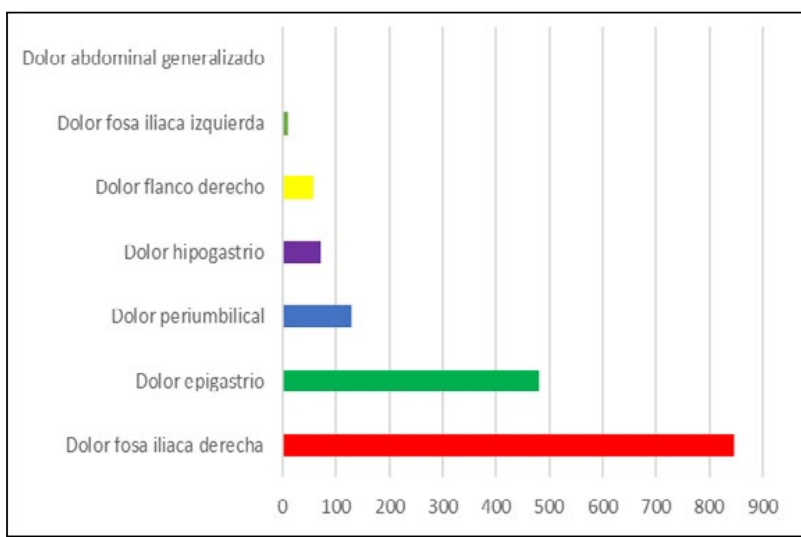

Figura 2. Motivos de Consultas más frecuentes- HCSL.

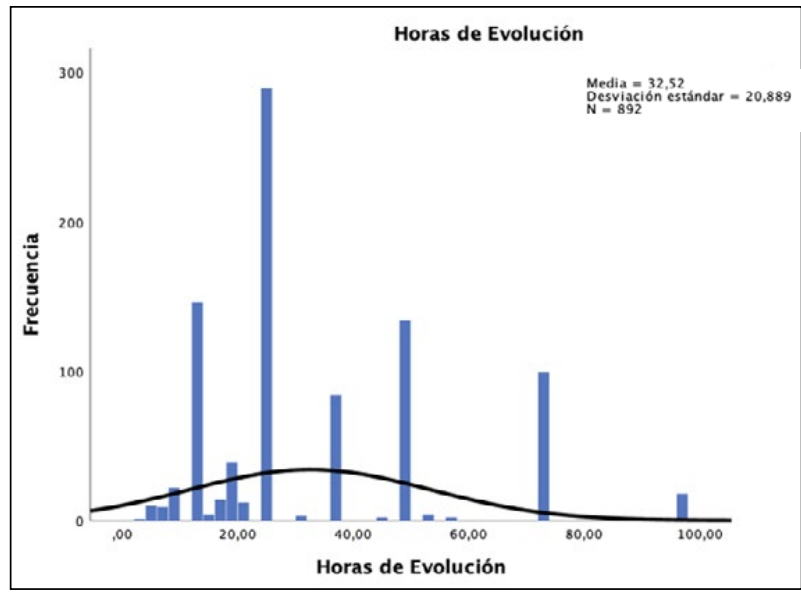

Figura 3. Distribución de las horas de evolución de la presentación de síntomas en los pacientes operados por apendicitis aguda entre los años 20152019 en el servicio de Urgencias Adultos de Cirugía del Hospital de Clínicas. 
Oblicua de Mc Burney, en el 13,9\% (124) la Transversa de Rocky Davis, en el 8,9\% (79) y el 8,5\% (76) se utilizó la Vía laparoscópica y la mediana infraumbilical, respectivamente.

La base de mayor frecuencia fue la sana con el 96\% (856) de los casos totales.

Los hallazgos quirúrgicos más frecuentes fueron la Gangrenosa con un 37,7\% (336) y la Flegmonosa con un 35,8\% (319), que sumados representan al 73,4\% (655) del total de cirugías. Los demás hallazgos en orden de frecuencia fueron; Congestiva con $17,6 \%$ (157), Necrótica con el 8,7\% (78) y Abdomen blanco con el 0,2\% (2) (Figura 5).

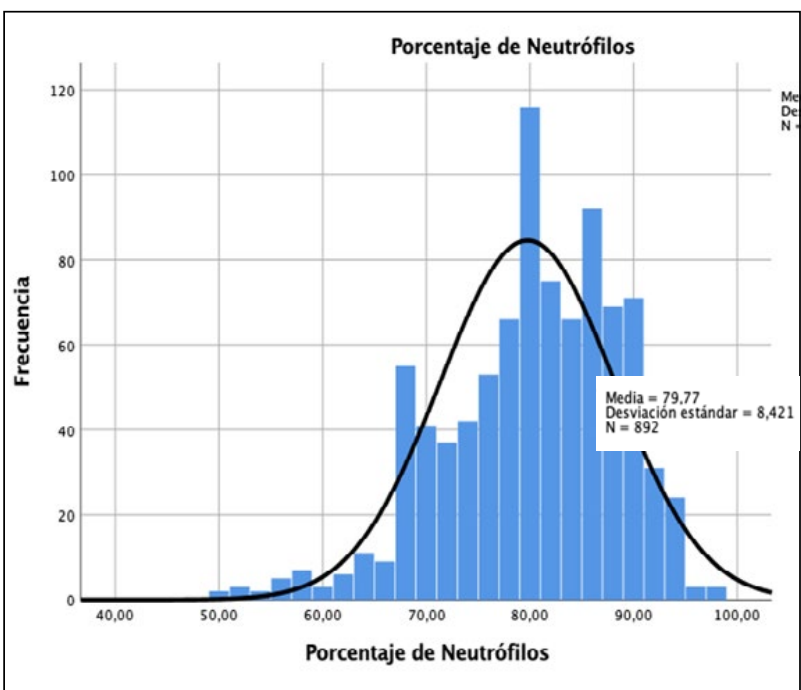

Figura 4. Distribución del porcentaje de neutrófilos observados en pacientes operados por apendicitis aguda entre los 2015-2019 en el servicio de Urgencias Adultos de Cirugía del Hospital de Clínicas.

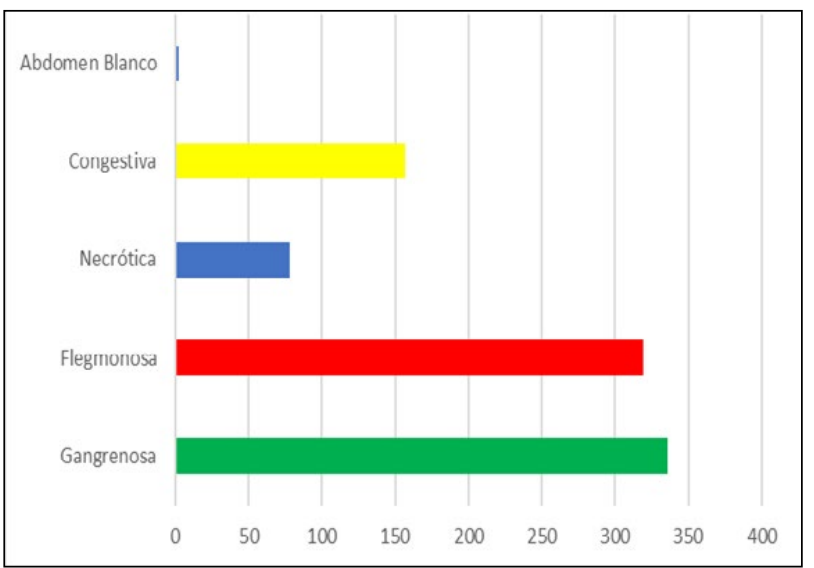

Figura 5. Pacientes operados por apendicitis aguda según hallazgos quirúrgicos encontrados entre los años 2015-2019 en el servicio de Urgencias Adultos de Cirugía del Hospital de Clínicas.

\section{DISCUSIÓN}

Trabajos similares que figuran en la literatura y reportes internacionales reportaron resultados similares, en nuestra revisión se obtuvo en un $77 \%$ una leucocitosis con neutrofilia siendo similares a los encontrados por otros autores, en donde estos hacen referencia incluso que el aumento de leucocitos es directamente proporcional al hallazgo intraoperatorio de apendicitis complicadas definiéndose como complicadas a las gangrenosas y perforadas ${ }^{(9)}$. Coincidimos en el hallazgo que evidenciamos mayor cantidad de casos de pacientes con apendicitis gangrenosas en un $37,7 \%$.

Nuestra revisión coincide con la evidencia en cuanto al sexo que con mayor frecuencia fue el sexo masculino, pero en cuanto al dolor en epigastrio como hallazgo inicial en la cronología de Murphy vemos en un 30,12\% comparando con otros autores como el Doctor Casado Méndez et al. que encontraron en un $50 \%{ }^{(10)}$.

El promedio en horas de evolución concordamos con la literatura utilizada con los medios y características demográficas y socioeconómicas parecidas a las nuestras en una media de $32 \mathrm{hs}$ frente a un 36 hs que reportan las revisiones internacionales.

En las nuevas actualizaciones de Guías en Tratamiento de Apendicitis Agudas recomiendan la vía laparoscópica como preferencia, en nuestro trabajo el hallazgo encontrado es del $8,5 \%$ resuelto por esta vía y los demás se realizaron por técnica abier$\mathrm{ta}^{11}$.Esto difiere con la literatura indexada, se puede deber a que en nuestro servicio de urgencias no tenemos disponibilidad de torre de laparoscopia a tiempo completo.

Los resultados encontrados impulsan a seguir por esta vía en beneficio de los pacientes en cuanto a seguridad en el diagnóstico y tratamiento de primera línea como es la laparoscópica.

\section{CONCLUSIÓN}

El desafío actual siempre para el cirujano es realizar un diagnóstico preciso y correcto para evitar conductas fallidas, por lo cual los métodos auxiliares juegan un rol importante para la toma de decisiones en situaciones difíciles ya que evidenciamos un $77 \%$ de presencia de leucocitosis en presencia de apendicitis agudas.

\section{REFERENCIAS BIBLIOGRÁFICAS}

1. Aade C, Benitez P, Aponte R. Historia deldiagnóstico y tratamiento de la apendicitis aguda. RFM, 2005, 28(1): 75-8.

2. Comité de elaboración de guias de práctica clínica. Guía de práctica clínica apendicitis aguda. Mexico. Oct 2014: 25

3. Hsien-Wei T, Jing-Tang W, Chien-Lung C, Shoei-Loong L, Min-Hsiung C. Modelo de decisión para el tratamiento de la apendicitis aguda con la tecnología de árbol de decisión: una modificación del sistema de puntuación de Alvarado. J Chin Med Assoc 2010; 73(8): 401-6.

4. Canavossoa L, Carenaa P, Carbonella J, Monjoa L, Palas C, Sánchez M et al. Dolor en fosa iliaca derecha y Scorede Alvarado. Cir Esp 2008; 83(5):247-51.

5. Barcat J. Sobre la apendicitis aguda: Amyand, Fitz, y unos pocos más. Med Bs Ars 2010; 70: 576-8.

6. Andersson M, Andersson R. El puntaje de respuesta inflamatoria apendicitis: una herramienta para el diagnóstico de apendicitis aguda que supera el puntaje de Alvarado. World J Surg (2008) 32: 843-1849.

7. Beltrán MA. et al. Rol del recuento de leucocitos y de la proteína C reactiva enniños con apendicitis. Revista Chilena de Cirugía 2007; 59 (1): $38-45$

8. Sanabria A. Tiempo de evolución de la apendicitis y riesgo de perforación. Rv. Colom Cir 2013; 28:24-30

9. Tesis de Grado para Titulo de Cirujano General Simblicia Cahuapaza Apaza.Índice de neutrofilos-linfocitos como predictorde apendicitis aguda complicada en el Hospital Regional de Ayacucho.Peru. 2016

10. Casado Méndez PR, Santos Fonseca RS, et al. Valor actual de la secuencia de Murphy en el diagnóstico de apendicitis aguda. AMC 2017; 21(1).

11. Di Salveiro S, Podda M, De Simone B, et al. Diagnosis and Treament of acute apendicitis: 2020 update of the WSES Jerusalen guidelines. World Journal Of Emerfency Surgery 2020. 